\title{
Influência das Alterações Hemodinâmicas Maternas sobre 0 Desenvolvimento Fetal
}

\author{
Effect of Maternal Hemodynamic Alterations on the Product of Conception
}

Vera Therezinha Medeiros Borges ${ }^{1}$, Beatriz B. Matsubara ${ }^{2}$, José Carlos Peraçoli ${ }^{1}$ Iracema Mattos Paranhos Calderon ${ }^{1}$, Joélcio Francisco Abbade ${ }^{1}$, Marilza Vieira Cunha Rudge ${ }^{1}$

\begin{abstract}
RESUMO
Objetivo: avaliar as alterações hemodinâmicas e estruturais cardiacas maternas nos três trimestres da gestação e relacioná-las com a classificação do recém-nascido, de acordo com o peso/idade gestacional.

Métodos: foi realizada avaliação ecocardiográfica em 22 gestantes, sem patologias, para estudo do débito cardíaco, pressão arterial média, diâmetro do átrio esquerdo e resistência periférica, em três periodos da gestação: antes da $12^{\underline{a}}$, na $26^{\underline{a}}$ e na $36^{\underline{a}}$ semanas de gestação. Dezessete gestantes deram à luz recém-nascidos com peso adequado, quatro, recém-nascidos pequenos, e uma gestante, recém-nascido grande para a idade gestacional.

Resultados: nas mães que deram à luz recém-nascidos pequenos para a idade gestacional, o débito cardiaco e o diâmetro do átrio esquerdo mantiveram-se inalterados, com tendência de elevação da pressão arterial média e aumento de $28 \%$ da resistência periférica, durante a gestação. As mães que deram à luz recém-nascidos adequados para idade gestacional tiveram aumento médio do débito cardíaco de $19 \%$ entre o primeiro e segundo trimestres $e$ de $8 \%$ entre o segundo e terceiro trimestres da gestação. O diâmetro do átrio esquerdo elevou-se próximo de $9 \%$ durante a gestação, com manutenção da pressão arterial média e tendência de queda da resistência periférica.

Conclusões: os resultados obtidos nesse trabalho suportam a associação entre adaptação hemodinâmica e peso do RN.
\end{abstract}

PALAVRAS-CHAVE: Gravidez normal. Adaptações cardiovasculares na gravidez. Resultado perinatal.

Introdução

As modificações hemodinâmicas e cardíacas que ocorrem durante a gestação guardam íntima relação com o resultado perinatal ${ }^{1}$. A hipervolemia fisiológica da gravidez garante o suporte de oxigênio e nutrientes para o feto e protege a mãe contra os efeitos danosos da diminuição do retorno venoso, decorrente da compressão uterina durante a gestação e da perda sangüínea no parto. Duvekot et al. ${ }^{2}$ demonstraram que ges-

\footnotetext{
1 Departamento de Ginecologia e Obstetricia e

2 Departamento de Clínica Médica da Faculdade de Medicina de Botucatu - UNESP

Correspondência:

Vera Therezinha Medeiros Borges

R: Vicente da Rocha Torres, 160 - Vila São Lúcio

18603-580 - Botucatu - SP
}

tantes com volemia inalterada ou pouco aumentada, no periodo da embriogênese, têm fetos com restrição de crescimento intra-útero. Em gestantes normais e em portadoras de hipertensão arterial crônica há nítida correlação entre a expansão do volume plasmático e o peso fetal ${ }^{3}$.

A hipervolemia parece ser diretamente proporcional ao número de fetos, embora a presença deste não seja essencial para a expansão da volemia, pois é detectada também na gestação molar. A adaptação hemodinâmica materna está diretamente relacionada com o tecido placentário, uma vez que está presente desde o início da gestação, quando o tecido placentário está no auge do seu desenvolvimento e declina na fase do envelhecimento placentário. O resultado da gravidez é dependente da qualidade da adaptação cardiovascular, que por sua vez é dirigida pelo feto 
e placenta ${ }^{1}$.

Em trabalho experimental com ratas espontaneamente hipertensas (SHR) mostrou-se que a restrição de crescimento intra-uterino decorreu da falta de aumento da volemia materna na pre$n h e z^{4}$. A sindrome de má adaptação do organismo materno à gravidez, descrita por Wallenburg 5 , caracterizada por falta de aumento do débito cardíaco e da volemia e não diminuição da resistência periférica e da reatividade vascular, está associada a recém-nascidos com restrição de crescimento intra-uterino.

Documentar as alterações hemodinâmicas circulatórias da gravidez e o momento em que ocorrem, por metodologia não-invasiva, é importante para se aprofundar os conhecimentos fisiológicos e compreender melhor as doenças induzidas pela gravidez ou coincidentes com a mesma. Essas alterações antecipam-se às demandas da gravidez, acontecendo, muitas vezes, antes de o ovo estar suficientemente desenvolvido para exigir grande suprimento ${ }^{6}$, e influenciam o peso do recém-nascido.

O objetivo deste estudo foi avaliar as alterações do débito cardíaco, pressão arterial média, diâmetro do átrio esquerdo e resistência periférica, nos três trimestres da gestação, relacionando-as com a classificação do recém-nascido de acordo com o peso/idade gestacional.

\section{Pacientes e Métodos}

O presente estudo é um ensaio clínico, prospectivo e longitudinal, em que foram selecionadas vinte e três gestantes no Serviço de Pré-Natal do Hospital das Clínicas da Faculdade de Medicina de Botucatu - UNESP. Foram critérios de inclusão: gestação única, idade gestacional menor que 12 semanas e ausência de patologias clínicas e obstétricas. A idade gestacional foi calculada pela regra de Naegele e confirmada por exame ultrasonográfico precoce.

A avaliação ecocardiográfica foi realizada em três momentos: $1^{\circ}$ trimestre (antes da 12 ${ }^{\mathrm{a}}$ semana), $2^{\circ}$ trimestre (26a semana) e $3^{\circ}$ trimestre (36 semana).

Logo após o nascimento, os recém-nascidos foram pesados e classificados em função do peso e da idade gestacional ${ }^{7} \mathrm{em}$ : PIG (pequeno para a idade gestacional), quando o peso ao nascer estava abaixo do percentil 10; AIG (adequado para a idade gestacional), quando o peso ao nascer estava entre o percentil 10 e 90 e GIG (grande para a idade gestacional), quando o peso ao nascer estava acima do percentil 90 .

\section{Exame ecocardiográfico}

Os exames foram realizados no período da manhã, entre $8 \mathrm{~h}$ e $11 \mathrm{~h}$, após repouso de $15 \mathrm{mi}$ nutos em ambiente calmo, com temperatura controlada, estando a paciente em decúbito lateral esquerdo e com a cabeça elevada a $15^{\circ}$. Foi utilizado o equipamento Sonos 2000 (Hewlett Packard), com transdutor de freqüência variável de 2,5 a 3,5 $\mathrm{MHz}$ e os registros foram efetuados em vídeo-printer da Sony. O procedimento seguiu as recomendações da "American Society of Echocardiography". Todas as medidas foram efetuadas em 3 ciclos cardiacos, tomando-se a média aritmética das aferições. Uma derivação eletrocardiográfica foi monitorada durante todo o exame.

\section{Modo bidimensional e Doppler}

As imagens bidimensionais foram obtidas nos cortes paraesternal (eixo menor e maior), e apical (quatro câmaras) com ou sem a valva aórtica, as quais foram analisadas segundo as recomendações da literatura ${ }^{8}$.

O diâmetro da aorta foi medido durante a sistole, no corte paraesternal eixo maior e correspondeu à maior distância entre as cúspides da valva aórtica. Esta medida foi utilizada para o cálculo do débito cardiaco. Foram analisados os fluxos pela valva aórtica por meio do Doppler contínuo e pelas valvas mitral, tricúspide e pulmonar usando o Doppler pulsátil.

O débito cardíaco foi calculado utilizando-se a fórmula: $\mathrm{DC}(\mathrm{L} / \mathrm{min})=\mathrm{VS}(\mathrm{L}) \times \mathrm{FC}$ (bat $/ \mathrm{min})$, onde DC é o débito cardíaco. Nesta fórmula, VS é o volume sistólico, que equivale à integral da velocidade do fluxo sistólico na aorta $(\mathrm{cm})$ multiplicado pela área de secção transversa do vaso $\left(\mathrm{cm}^{2}\right)$, e FC é a freqüência cardíaca.

A resistência vascular periférica foi estimada aplicando-se a seguinte fórmula: Rp (dyn/s/ $\left.\mathrm{cm}^{5}\right)=$ PAM $(\mathrm{mmHg}) \times 80 / \mathrm{DC}(\mathrm{L} / \mathrm{min})$, onde PAM é a pressão arterial média. A PAM foi calculada pela fórmula $=\mathrm{PD}+1 / 3$ (PS $-\mathrm{PD})$.

\section{Modo M}

A imagem bidimensional do ventrículo esquerdo foi obtida com o transdutor colocado no $3^{\circ}$ ou 4을 espaço intercostal esquerdo, no corte eixo menor. O cursor modo $\mathrm{M}$ foi alinhado na cavidade ventricular entre os dois músculos papilares para registro da imagem monodimensional da câmara. A imagem monodimensional do átrio esquerdo foi obtida a partir do mesmo corte, ao nivel da valva aórtica.

Imediatamente após o registro ecocardiográfico, estando a paciente na mesma posição, foi 
aferida a pressão arterial, no braço esquerdo, por meio do esfigmomanômetro de coluna de mercúrio. Para aferição da pressão sistólica e diastólica

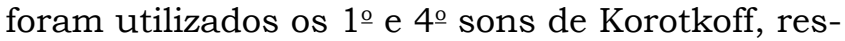
pectivamente. Foram efetuadas três aferições e tomou-se a média aritmética como resultado final. As aferições da pressão arterial foram realizadas pela mesma pessoa que realizou os exames ecocardiográficos.

As comparações estatísticas entre os dois grupos foram realizadas pelo teste de Fisher. As diferenças entre os grupos foram consideradas significantes se $\mathrm{p}<0,05$.

O estudo foi previamente aprovado pelo Comitê de Ética em Pesquisa da Faculdade de Medicina de Botucatu - UNESP. O consentimento esclarecido foi assinado por todas as gestantes que participaram do estudo.

\section{Resultados}

Dezessete gestantes deram à luz recémnascidos AIG, quatro, recém-nascidos PIG, e uma, recém-nascido GIG, que foi excluída por se tratar de um único caso.

Na Tabela 1, estão as características maternas e o peso médio dos recém-nascidos dos dois grupos estudados. A idade materna, paridade, estatura e idade gestacional ao nascimento são similares entre os grupos. O peso dos recém-nascidos classificados como adequados para a idade gestacional foi significativamente maior (3.334 g) do que os dos classificados como pequenos para a idade gestacional (2.376 g).

Tabela 1 - Média e desvio-padrão das características maternas e do peso dos recémnascidos segundo a classificação dos RNs em adequado (AIG) e pequenos (PIG) para a idade gestacional.

\begin{tabular}{lcc}
\hline & AIG & PIG \\
\hline Idade materna (anos) & $24.9 \pm 6.5$ & $22.0 \pm 6.30$ \\
Paridade & $1.24 \pm 0.44$ & $1.75 \pm 1.50$ \\
Estatura materna $(\mathrm{m})$ & $1.55 \pm 0.48$ & $1.55 \pm 0.082$ \\
IG no momento do parto & $39.84 \pm 1.26$ & $38.68 \pm 2.14$ \\
Peso do RN (g) & $3.334 \pm 351$ & $2.376 \pm 337^{\star}$ \\
\hline
\end{tabular}

${ }^{*} p<0,01$

Nos recém-nascidos de peso pequeno para a idade gestacional (PIG), o débito cardiaco e o diâmetro do átrio esquerdo materno mantiveram-se inalterados no decorrer da gestação. Embora não tenha havido diferença significante, podemos observar tendência de elevação da pressão arterial média durante a evolução da gestação, e aumento de $28 \%$ da resistência periférica entre o $2^{\circ}$ e $3^{\circ}$ trimestres nessas gestantes.

Nas mães que deram à luz recém-nascidos de peso adequado para idade gestacional (AIG) houve aumento médio do débito cardiaco de $19,2 \%$ entre o primeiro e segundo trimestres e de $7,74 \%$ entre o segundo e terceiro trimestres da gestação. O diâmetro do átrio esquerdo elevou-se próximo de $9 \%$ durante a gestação, com manutenção da pressão arterial média e tendência de queda da resistência periférica (Figuras $1,2,3,4)$.

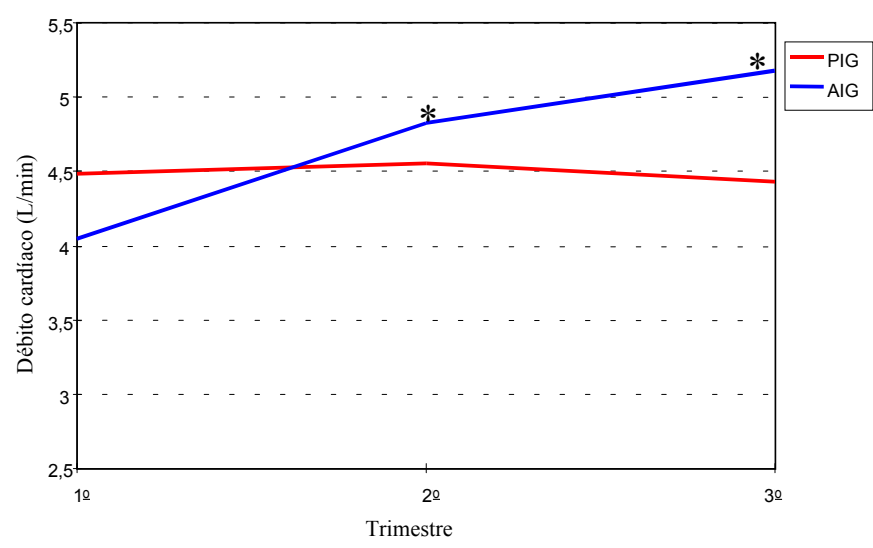

${ }^{*} p<0,05$ vs 10 trimestre

Figura 1 - Média do débito cardíaco nos três trimestres da gestação de mães de recémnascidos pequenos (PIG) e adequados (AIG) para a idade gestacional.

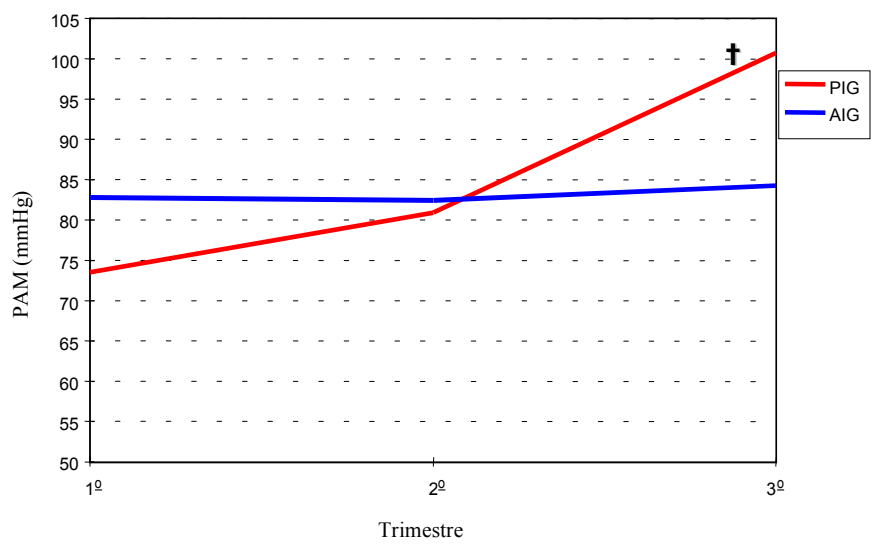

t $p=0,07$ vs 10 trimestre

Figura 2 - Média da pressão arterial média (PAM) nos três trimestres da gestação de mães de recém-nascidos pequenos (PIG) e adequados (AIG) para a idade gestacional. 


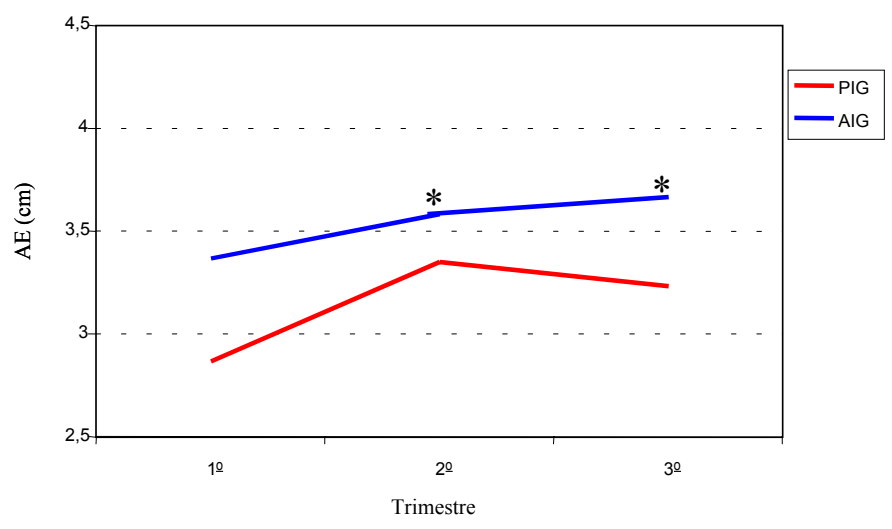

${ }^{*} p<0,05$ vs 10 trimestre

Figura 3 - Média do diâmetro do átrio esquerdo (AE) nos três trimestres da gestação de mães de recém-nascidos pequenos (PIG) e adequados (AIG) para a idade gestacional.

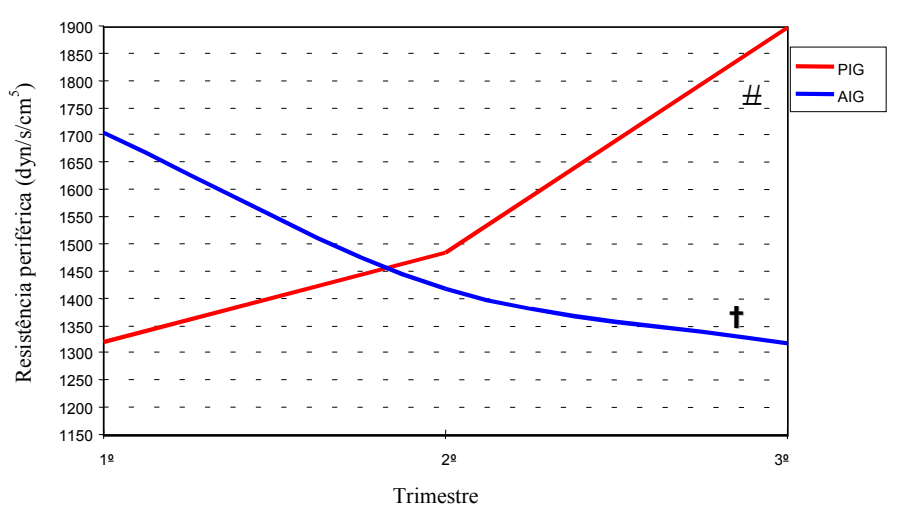

$\# p<0,05$ vs 2 o trimestre

$+p=0,06$ vs 10 trimestre

Figura 4 - Média da resistência periférica nos três trimestres de gestação de mães de recém-nascidos pequenos (PIG) e adequados (AIG) para a idade gestacional.

\section{Discussão}

As modificações hemodinâmicas e cardíacas que ocorrem na gestação guardam intima relação com o resultado perinatal ${ }^{1,5}$. Para avaliar essas alterações a metodologia mais utilizada é a ecocardiografia, por ser método não-invasivo e reproduzivel em vários momentos. A fim de otimizar a reprodutibilidade, padronizou-se, neste trabalho, que cada medida fosse repetida três vezes, adotando-se o respectivo valor médio ${ }^{9}$. Além disso, todos os procedimentos foram efetuados por um único examinador.

Há nítida correlação entre a expansão da volemia e o peso fetal nas gestantes sem patologias e nas portadoras de hipertensão arterial crôni$\mathrm{ca}^{3}$. Alguns autores encontraram baixa volemia em gestantes com restrição de crescimento intrauterino ${ }^{10,11}$. Além disso, trabalhos experimentais com animais reforçam a hipótese de que a fisiopatologia da restrição de crescimento intrauterino decorre da ausência de expansão da volemia $^{12,13}$.

O diâmetro do átrio esquerdo reflete aumento do volume que retorna ao coração, sendo indicador indireto do aumento de volemia. Como o aumento do tamanho do átrio esquerdo guarda relação com a volemia materna, procurou-se relacioná-lo com o resultado perinatal. A medida do átrio esquerdo das mães dos recém-nascidos PIG foi sempre inferior à das mães dos recém-nascidos AIG, demonstrando indiretamente a deficiência na expansão da volemia. Esse resultado é concordante com estudos prévios que utilizaram diferentes técnicas ${ }^{2}$.

Rosso et al. ${ }^{11}$ demonstraram que a ausência da expansão da volemia, próximo ao termo, está associada à diminuição do débito cardíaco, levando à redução do fluxo sanguíneo uterino, causando a restrição de crescimento intrauterino. Duvekot et al. ${ }^{14}$ observaram falha no aumento do débito cardíaco logo nas primeiras semanas de gestação nas gestantes com restrição de crescimento intra-uterino.

Os nossos resultados mostraram que as mães dos recém-nascidos PIG não apresentaram alterações no débito cardíaco com a evolução da gestação, enquanto que nas mães dos recém-nascidos AIG houve aumento durante toda a gestação.

Os mecanismos que desencadeiam a expansão da volemia nas gestações sem patologias ainda não são muito bem compreendidos. Sabe-se que o mecanismo-chave é a ativação do sistema renina-angiotensina-aldosterona (SRAA), que causa retenção de sal e água e, conseqüentemente, expansão da volemia, porém a causa desta ativação é desconhecida. Dados sugerem que estrógeno e ou vasodilatação arterial podem ser os responsáveis ${ }^{15}$. Salas e Rosso ${ }^{10}$ encontraram redução dos niveis de renina, angiotensina e aldosterona em gestantes com restrição de crescimento intrauterino, sugerindo que uma vasodilatação inadequada falhou na ativação do SRAA.

Seguindo esse raciocínio, alguns autores encontraram aumento da resistência periférica em gestantes com fetos com restrição de crescimento intra-uterino ${ }^{2,11}$. Em nosso estudo, os comportamentos da resistência periférica e da pressão arterial média foram diferentes, quando a gestação resultou em recém-nascido PIG ou AIG. As mães de recém-nascidos PIG, apesar de normotensas durante todo o ciclo gravídico, apresentaram tendência à elevação da pressão arterial média e aumento da resistência periférica. Isto nos leva a supor que, nessas gestantes, a alteração mínima do débito cardíaco decorreu do aumento 
da resistência periférica, que ocasionou elevação da pressão arterial média, permitindo-nos a dizer que ocorreu a síndrome da má adaptação do organismo materno ${ }^{5}$.

Quando o resultado foi um recém-nascido de peso adequado, a pressão arterial média manteve-se constante e a resistência periférica diminuiu. Tais achados reforçam o conceito de que a adequada adaptação do organismo materno à gravidez é fundamental para o resultado perinatal ideal $^{1}$.

Os resultados obtidos nesse trabalho evidenciam que a adaptação hemodinâmica é necessária para o bom desenvolvimento do concepto.

\section{SUMMARY}

Purpose: to evaluate maternal hemodynamic and cardiac structural changes during the three trimesters of pregnancy and to relate them to the weight/gestational age of the newborn.

Methods: twenty-two healthy pregnant women were submitted to echocardiography for the study of cardiac output, mean arterial pressure, left atrium diameter, and peripheral resistance during three periods of pregnancy, i.e., before the 12th week and at the 26th and 36th weeks of pregnancy. Seventeen pregnant women gave birth to infants with adequate weight for gestational age, four gave birth to small for gestational age newborns and one gave birth to a large for gestational age infant.

Results: among mothers of low weight newborns, cardiac output and left atrium diameter remained constant, mean arterial pressure showed a tendency to increase and peripheral resistance was significantly increased (28\%), during the gestation. Among the mothers of adequate weight newborns there was an increase in cardiac output of $19 \%$ in the second trimester and $8 \%$ in the third. The left atrial diameter increased approximately 9\% during the gestation, with maintenance of mean arterial pressure and a tendency to a decrease in peripheral resistance.

Conclusion: the present results support an association between hemodynamic adaptation and weight newborn.

KEY WORDS: Pregnancy, normal. Cardiovascular adaptations in pregnancy. Perinatal outcome.

\section{Referências}

1. Cunningham FG, Macdonald PC, Gant NF, Leveno KJ, Gilstrap LC $3^{\text {rd }}$. Williams obstetrics. $20^{\text {th }}$ ed. Stamford: Appleton \& Lange; 1997. The placenta and fetal membranes; p.95-123.
2. Duvekot JJ, Cheriex EC, Pieters FA, Peeters LL. Severely impaired fetal growth is preceded by maternal hemodynamic maladaptation in very early pregnancy. Acta Obstet Gynecol Scand 1995; 74:693-7.

3. Gallery ED, Brown MA. Control of sodium excretion in human pregnancy. Am J Kidney Dis 1987; 9:290-5.

4. Peraçoli JC. Hipertensão arterial e gravidez: estudo experimental em ratas espontaneamente hipertensas (SHR) [tese]. Botucatu: Universidade Estadual Paulista; 1990.

5. Wallenburg HCS. Hemodynamics in hypertensive pregnancy. In: Rubin PC, editor. Hypertension in Pregnancy. $1^{\text {st }}$ ed. Amsterdam: Elsevier; 1988. p.66-101.

6. Rezende J. Obstetrícia. 6. ed. Rio de Janeiro: Guanabara-Koogan; 1990. p.1117.

7. Fescina RH, Schwarcz R, Díaz AG. Vigilancia del crescimento fetal. Manual de autoinstruccion. $1^{\text {st }}$ ed. Montevideo, Clap, 1992. Publ. Cient., 1261.

8. Henry WL, De Maria AN, Gramiak R, et al. Report of the American Society of Echocardiography. Committee on nomenclature in two dimensional echocardiography. Circulation 1980; 62:212-5.

9. Van Oppen AC, Stigter RH, Bruinse HW. Cardiac output in normal pregnancy: a critical review. Obstet Gynecol 1996; 87:310-8.

10.Salas SP, Rosso P. Plasma volume, renal function, and hormonal levels in pregnant women with idiopathic fetal growth restriction or preeclampsia. Hypertens Pregnancy 1989; 17:6979.

11.Rosso P, Donoso E, Braun S, Espinosa R, Fernandez C, Salas SP. Maternal hemodynamic adjustments in idiopathic fetal growth retardation. Gynecol Obstet Invest 1993; 35:162-5.

12.Rosso P, Kava R. Effects of food restriction on cardiac output and blood flow to the uterus and placenta in pregnant rat. J Nutr 1980; 110:23504.

13.Daniel SS, James S, Stark RI, Trooper PJ. Prevention of the normal expansion of maternal plasma volume: a model for chronic fetal hypoxaemia. $\mathrm{J}$ Dev Physiol 1989; 11:225-33.

14.Duvekot JJ, Cheriex EC, Pieters FA, Menheere PP, Peeters LL. Early pregnancy changes in hemodynamics and volume homeostasis are consecutive adjustments triggered by a primary fall in systemic vascular tone. Am J Obstet Gynecol 1993; 169:1382-92.

15.Schrier RW, Briner VA. Peripheral arterial vasodilation hypothesis of sodium and water retention in pregnancy: implications for pathogenesis of preeclampsia-eclampsia. Obstet Gynecol 1991; 77:632-9. 